\title{
The nitric oxide: A molecule of possible therapeutic use against aquatic stress in fishes.
}

\section{Singh A*}

Department of Zoology, R.K. Talreja College of Arts, Science and Commerce, University of Mumbai, Maharashtra, India

\section{Editorial}

In several toxicological research studies involving fish as animal model, it has been seen that fish die primarily because of suffocation caused due to dismantling of the vascular components followed by wear and tear and sloughing of epithelium layer of the respiratory organs such as gills and accessory respiratory organs (ARO) especially the air-breathing organs (ABO) found in some air-breathing fish species like Clarias batrachus, Clarias gariepinus, Anabas sp. etc. The gills are main respiratory organs and are fully aquatic in respiratory function while the $\mathrm{ABO}$, on the other hand, are said to be aerial in nature i.e., extract the oxygen from atmospheric air. The $\mathrm{ABO}$ is hence more important for air-breathing fishes as they enable them to survive even in a condition of stress produced by ambient water contaminants/pollutants. The gills, apart from the role in breathing, also assist the fish in osmoregulation, in maintaining acid-base balance and in getting rid of nitrogenous waste materials produced in various metabolic reactions. Both gills and $\mathrm{ABO}$, as they purely reside in suprabranchial chamber of either lateral side of fish head and always remain in direct touch with external milieu are more vulnerable and are probably affected first when the fish is exposed to any stressors/ contaminants in aquatic bodies/reservoirs. The researchers have found that the fish, very often, try to compensate adverse water quality conditions by flowing more and more blood in blood capillaries and subepithelial blood vessels of the gills and ABO. Thus, the overall efforts made by the fish create an extra pressure on these delicate organs which ultimately results in histomorphological manifestations including wear and tear, dismantling of ladder like vascular components and sloughing of surface epithelium leading to haemorrhage and ultimately death of the fish. From research studies, it is clear that the responses of these two vital organs towards aquatic stressors/ contaminants are almost compensatory in nature and perhaps in the direction to minimise and/or neutralise the effects caused for at least temporarily. This also corroborates the common origin and similar anatomical patterns of the gills and $\mathrm{ABO}$ as evidenced in several researches.

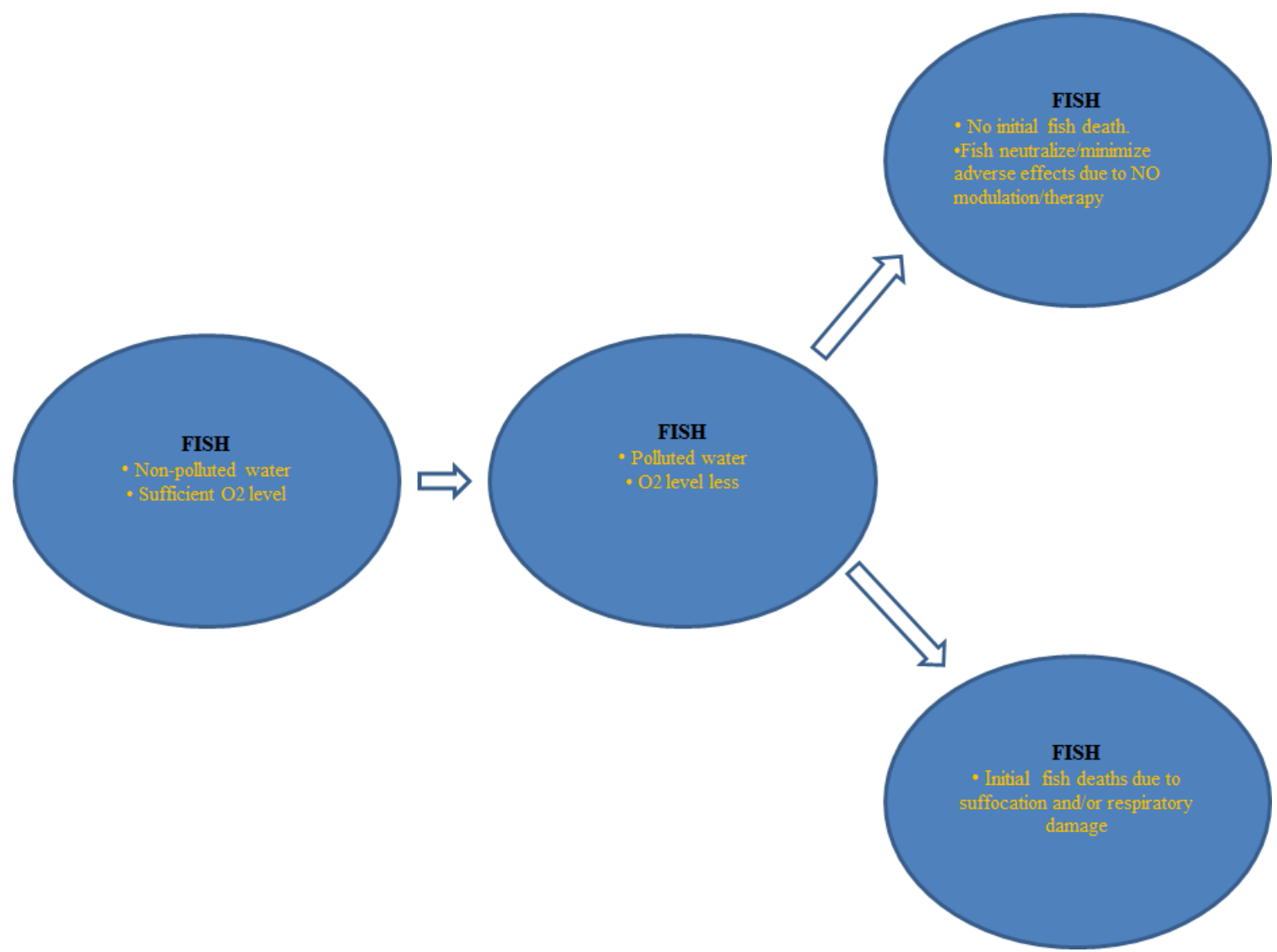

Figure 1. A schematic representation indicating how the fishes will neutralize/minimize the effects of pollution and survive for longer time when supplemented with $\mathrm{NO}$. 
The Nitric Oxide (NO) detection in animal tissues and elucidation of some of its biological role, in past few years, has opened a new rationale for the biologists to do researches in the field of biological sciences and especially the animal sciences. In several research studies on mammals and other animals including the fishes, it has been confirmed that NO play very important role in many physiological functions including neurotransmission and vasodilation. NO is a short lived gaseous molecule and originates endogenously from oxidation of $\mathrm{L}$-arginine to L-citruline in response to receptor stimulation and under catalytic control of Nitric Oxide Synthase (NOS) enzyme. There are three isomers of NOS namely neuronal NOS (nNOS), endothelial NOS (eNOS) and inducible NOS (iNOS) which require nicotinamide adenine dinucleotide phosphate (NADPH) as cofactor for generation of NO from L-arginine, a substrate molecule for NO production in animal tissues/organs. In cardiovascular system of the animals, during vasodilation, it is established that NO is released as a part of adaptive mechanism and enables blood vessels and hence the animal to respond the changes in surrounding by regulating the blood flow and maintaining the blood pressure through an action on the smooth muscles of the vessels. In studies on some fishes, the presence of NO synthesizing enzyme (NOS) in the gills has been evidenced. The role of NO as vasodilator and more specifically, if I am stating, stress (aquatic) reliever in fishes is however a matter of great investigation. To elaborate and know more about the role of NOS and hence the NO in gills and ABO of the fish, the work has been initiated at Postgraduate Department of Zoology, R.K. Talreja College of Arts, Science and Commerce, Ulhasnagar-3, Maharashtra, India in the form of a small Research Project (Minor Research Project) sanctioned from University Grants Commission, Govt. of India, New Delhi, India.

The findings of Research Project will not only help the Ichthyologists to understand, how the life of fishes can be saved for longer time in such an era of huge industrialization and urbanization and other human activities which are all together life threatening to the fishes but also the Toxicologists to trace out the possible pathways involved in vasodilatation process (Figure 1).

\section{*Correspondence to:}

Singh A

Department of Zoology

R.K. Talreja College of Arts, Science and Commerce University of Mumbai

Maharashtra, India

Tel: 02512730297

E-mail: aksrktzool@yahoo.in 